\title{
PURE STATES, POSITIVE MATRIX POLYNOMIALS AND SUMS OF HERMITIAN SQUARES
}

\author{
IGOR KLEP AND MARKUS SCHWEIGHOFER
}

\begin{abstract}
Let $M$ be an archimedean quadratic module of real $t \times t$ matrix polynomials in $n$ variables, and let $S \subseteq \mathbb{R}^{n}$ be the set of all points where each element of $M$ is positive semidefinite. Our key finding is a natural bijection between the set of pure states of $M$ and $S \times \mathbb{P}^{t-1}(\mathbb{R})$. This leads us to conceptual proofs of positivity certificates for matrix polynomials, including the recent seminal result of Hol and Scherer: If a symmetric matrix polynomial is positive definite on $S$, then it belongs to $M$. We also discuss what happens for nonsymmetric matrix polynomials or in the absence of the archimedean assumption, and review some of the related classical results. The methods employed are both algebraic and functional analytic.
\end{abstract}

\section{INTRODUCTION}

We write $\mathbb{N}:=\{1,2, \ldots\}, \mathbb{Q}, \mathbb{R}$ and $\mathbb{C}$ for the sets of natural, rational, real and complex numbers, respectively. The complex numbers $\mathbb{C}$ always come equipped with the complex-conjugation involution. For any matrix $A$ over a ring with involution $\mathcal{A}$, we denote by $A^{*}$ its conjugate transpose. If $A$ is a real matrix, $A^{*}$ is simply its transpose. Let $\operatorname{Sym} \mathcal{A}^{t \times t}:=\left\{A \in \mathcal{A}^{t \times t} \mid A=A^{*}\right\}$ be the set of all symmetric $t \times t$ matrices. Examples of these include hermitian squares, i.e., elements of the form $A^{*} A$ for some $A \in \mathcal{A}^{t \times t}$.

Recall that a matrix $A \in \mathbb{R}^{t \times t}$ is called positive semidefinite if it is symmetric and $\langle A v, v\rangle=v^{*} A v \geq 0$ for all vectors $v \in \mathbb{R}^{t}, A$ is positive definite if it is positive semidefinite and invertible, and is called negative semidefinite if $-A$ is positive semidefinite. For matrices $A$ and $B$ of the same size, we write $A \preceq B$ (respectively $A \prec B$ ) to express that $B-A$ is positive semidefinite (respectively positive definite). Geometrically, $A \in \operatorname{Sym} \mathbb{R}^{t \times t}$ is positive semidefinite if and only if all of its eigenvalues are nonnegative, $A$ is positive definite if and only if all of its eigenvalues are positive, and $A$ is not negative semidefinite if and only if one of its eigenvalues is positive. The following algebraic characterizations are easy to prove:

Proposition 1. Let $A \in \operatorname{Sym} \mathbb{R}^{t \times t}$.

(a) $A \succeq 0$ if and only if $A$ is a sum of hermitian squares in $\mathbb{R}^{t \times t}$;

Date: September 29, 2009.

2000 Mathematics Subject Classification. Primary 15A48, 11E25, 13J30; Secondary 15A54, 14P10, 46A55.

Key words and phrases. matrix polynomial, pure state, positive semidefinite matrix, sum of hermitian squares, Positivstellensatz, archimedean quadratic module, Choquet theory.

Both authors were supported by the French-Slovene partnership project Proteus 20208ZM. 
(b) $A \npreceq 0$ if and only if there exist $B_{i}, C_{j} \in \mathbb{R}^{t \times t}$ such that

$$
\sum_{i} B_{i}^{*} A B_{i}=1+\sum_{j} C_{j}^{*} C_{j}
$$

The main goal of this article is to explain how this proposition extends to matrix polynomials, i.e., elements of the ring $\mathbb{R}[\bar{X}]^{t \times t}$ where $\mathbb{R}[\bar{X}]$ is the ring of polynomials in $n$ variables $\bar{X}=\left(X_{1}, \ldots, X_{n}\right)$ with coefficients from $\mathbb{R}$. Note that in $\mathbb{R}^{t \times t}$, every sum of hermitian squares is of course a hermitian square. The reason why we speak of sums of hermitian squares in Proposition 1 is that this is no longer true in $\mathbb{R}[\bar{X}]^{t \times t}$. Note however, that $A \in \mathbb{R}[\bar{X}]^{t \times t}$ is a sum of hermitian squares in $\mathbb{R}[\bar{X}]^{t \times t}$ if and only if there is $u \in \mathbb{N}$ and $B \in \mathbb{R}[\bar{X}]^{u \times t}$ such that $A=B^{*} B$.

Let $\mathcal{A}$ be a ring with involution $a \mapsto a^{*}$ (i.e., $(a+b)^{*}=a^{*}+b^{*},(a b)^{*}=b^{*} a^{*}$ and $a^{* *}=a$ for $\left.a, b \in \mathcal{A}\right)$ and set $\operatorname{Sym} \mathcal{A}:=\left\{a \in \mathcal{A} \mid a=a^{*}\right\}$. A subset $M \subseteq \operatorname{Sym} \mathcal{A}$ is called a quadratic module in $\mathcal{A}$ if

$$
1 \in M, \quad M+M \subseteq M \text { and } a^{*} M a \subseteq M \text { for all } a \in \mathcal{A} .
$$

To every $G \subseteq \operatorname{Sym} \mathbb{R}[\bar{X}]^{t \times t}$, we associate the set

$$
S_{G}:=\left\{x \in \mathbb{R}^{n} \mid \forall g \in G: g(x) \succeq 0\right\}
$$

and the quadratic module $M_{G}$ generated by $G$ in $\mathbb{R}[\bar{X}]^{t \times t}$. That is,

$$
M_{G}=\left\{\sum_{i=1}^{N} p_{i}^{*} g_{i} p_{i} \mid N \in \mathbb{N}, g_{i} \in\{1\} \cup G, p_{i} \in \mathbb{R}[\bar{X}]^{t \times t}\right\} .
$$

In particular, $M_{\varnothing}$ is the set of all sums of hermitian squares in $\mathbb{R}[\bar{X}]^{t \times t}$.

Given a matrix polynomial $f \in \operatorname{Sym} \mathbb{R}[\bar{X}]^{t \times t}$ and $S \subseteq \mathbb{R}^{n}$, we write $f \succeq 0$ on $S$ if for all $x \in S, f(x) \succeq 0$. Likewise we use $f \succ 0, f \npreceq 0$. With this notation, $f \in M_{G}$ implies $f \succeq 0$ on $S_{G}$.

In the sequel, we investigate how $M_{G}$ can be used to describe matrix polynomials $f \in \operatorname{Sym} \mathbb{R}[\bar{X}]^{t \times t}$ with $f \succ 0, f \succeq 0$ or $f \npreceq 0$ on $S_{G}$. In Section 2 , the case $G=\varnothing$ is considered; classical results on globally positive semidefinite matrix polynomials in one or more variables are reviewed, and then we turn to nowhere negative semidefiniteness. We give a sum of hermitian squares representation with denominators in the one variable case and prove mostly negative results for the case of matrix polynomials in several variables.

Our main results are presented in Section 3 which is devoted to the case of compact $S_{G}$. Actually we work under the slightly stronger assumption that the quadratic module $M_{G}$ is archimedean (which can be enforced by possibly enlarging $G$ without changing $S_{G}$ ). Under this assumption we describe all pure states on $\operatorname{Sym} \mathbb{R}[\bar{X}]^{t \times t}$ (extremal linear forms positive with respect to $M_{G}$ ) as being of the form $p \mapsto\langle p(x) v, v\rangle$ for some $x \in S_{G}$ and $v \in \mathbb{R}^{t}$. From this we deduce certificates, in the spirit of Proposition 1, for matrix polynomials being nowhere negative semidefinite on $S_{G}$ or positive semidefinite on $S_{G}$ in the spirit of Proposition 1. The latter was originally proved by Hol and Scherer [HS] with entirely different methods. 
For a study of positivity of matrix polynomials in noncommuting variables, we refer the reader to Helton and McCullough, see e.g. [HM]. Burgdorf, Scheiderer and the second author [BSS] investigate pure states and their role in commutative algebra.

\section{Globally positive matrix polynomials}

There are various notions of positivity for matrices. Like in Proposition 1, we consider positivity of the smallest and largest eigenvalue, respectively.

2.1. Globally positive semidefinite matrix polynomials. By Gauß' theorem, every nonnegative univariate real polynomial is a sum of two squares of real polynomials. The extension to univariate real matrix polynomials was first given by Jakubovič J Ja and is in a different form commonly known as the Kalman-Jakubovič-Popov lemma AIP. It is one of the vast number of matrix factorization results obtained and used in operator and control theory GKS, GLR, RR]. We refer the reader to [AIP for a nice algorithmic proof; see also Dj1].

Theorem 2 (Jakubovič). For $f \in \operatorname{Sym} \mathbb{R}[Z]^{t \times t}$, the following are equivalent:

(i) $f \succeq 0$ on $\mathbb{R}$;

(ii) $f$ is a sum of two hermitian squares in $\mathbb{R}[Z]^{t \times t}$.

Note that (ii) is equivalent to $f=g^{*} g$ for some $g \in \mathbb{R}[Z]^{2 t \times t}$, or $f=\sum_{i=1}^{2 t} v_{i} v_{i}^{*}$ for some $v_{i} \in \mathbb{R}[Z]^{t}$ (cf. also [CLR, [FRS]).

The multivariate version of Gauß' theorem is Artin's solution to Hilbert's 17th problem [Ma, $\mathrm{PD}$ ]: a nonnegative multivariate real polynomial is a sum of squares of real rational functions. A multivariate version of Jakubovič's theorem (and at the same time the matrix version of Artin's theorem) was obtained by Gondard and Ribenboim GR in 1974 and reproved several times, e.g. Dj2, $\mathrm{PS}, \mathrm{HiN}$.

Theorem 3 (Gondard \& Ribenboim). For $f \in \operatorname{Sym} \mathbb{R}[\bar{X}]^{t \times t}$, the following are equivalent:

(i) $f \succeq 0$ on $\mathbb{R}^{n}$;

(ii) $p^{2} \bar{f}$ is a sum of hermitian squares in $\mathbb{R}[\bar{X}]^{t \times t}$ for some nonzero $p \in \mathbb{R}[\bar{X}]$.

Proof. From (ii) it follows that $f \succeq 0$ on $\left\{x \in \mathbb{R}^{n} \mid p(x) \neq 0\right\}$ and hence (i). Conversely, suppose that (i) holds. By diagonalization of quadratic forms over a field, there exists an invertible matrix $g \in \mathbb{R}(\bar{X})^{t \times t}$ and a diagonal matrix $d \in \mathbb{R}(\bar{X})^{t \times t}$ such that $f=g^{*} d g$. By (i), $d$ is positive semidefinite where defined. By Artin's solution to Hilbert's 17th problem, we find a nonzero $p \in \mathbb{R}[\bar{X}]$ such that $p^{2} d$ is a sum of (hermitian) squares in $\mathbb{R}[\bar{X}]^{t \times t}$. Without loss of generality, we can assume that $p^{2}$ also clears the denominators in $g$.

In the literature cited above, one can find refinements of (ii) at the expense of more complicated proofs, e.g. (ii') $p^{2} f$ is a sum of squares in the commutative ring $\mathbb{R}[\bar{X}, f] \subseteq \mathbb{R}[\bar{X}]^{t \times t}$ for some nonzero $p \in \mathbb{R}[\bar{X}]$. Also, Gondard and Ribenboim [GR] prove a bound on the number of hermitian squares needed. 
2.2. Nowhere negative semidefinite matrix polynomials. We now turn to symmetric nonnegative matrix polynomials which are not negative semidefinite globally, i.e., whose evaluations all have at least one positive eigenvalue. We start by giving an analog of Proposition 1(b) for univariate matrix polynomials. Though this is the perfect counterpart to the well known Theorem 2, it is to the best of our knowledge an entirely new result.

Theorem 4. For $f \in \operatorname{Sym} \mathbb{R}[Z]^{t \times t}$, the following are equivalent:

(i) $f \npreceq 0$ on $\mathbb{R}$;

(ii) there exist $p_{i} \in \mathbb{R}[Z]^{t \times t}$ such that $\sum_{i} p_{i}^{*} f p_{i}-1$ is a sum of hermitian squares.

Proof. It is clear that (ii) $\Rightarrow$ (i), cf. Proposition 1(b).

To prove the converse, suppose first that $f$ is diagonal, say $f=\left[\begin{array}{lll}f_{1} & & \\ & \ddots & \\ & & f_{t}\end{array}\right]$. By assumption (i), $S_{\left\{-f_{1}, \ldots,-f_{t}\right\}}=\varnothing$ is compact. Since we are in the univariate case, this implies that the quadratic module $M_{\left\{-f_{1}, \ldots,-f_{t}\right\}} \subseteq \mathbb{R}[Z]$ contains all polynomials positive on $S_{\left\{-f_{1}, \ldots,-f_{t}\right\}}$ [PD, Theorem 6.3.8]. In particular, with $f_{0}:=-1$, there are $g_{i j} \in \mathbb{R}[Z]$ satisfying

$$
-1=\sum_{i=0}^{t}\left(-f_{i}\right) \sum_{j} g_{i j}^{2}
$$

Observe that for each $i$,

$$
f_{i}=\sum_{k=1}^{t} E_{i k}^{*} f E_{i k} \in M_{\{f\}}
$$

where $E_{j k}$ are the $t \times t$ matrix units. Thus (1) implies

$$
\sum_{i, k=1}^{t} \sum_{j}\left(E_{i k} g_{i j}\right)^{*} f\left(E_{i k} g_{i j}\right)-1=\sum_{i=1}^{t} \sum_{j} g_{i j}^{*} f_{i} g_{i j}-1=\sum_{j} g_{0 j}^{2}
$$

is a sum of hermitian squares.

Now suppose $f$ is not necessarily diagonal. By the version of the LDU decomposition for matrix polynomials given in [Sm2, Proposition 8], there exist diagonal matrices $D_{\ell} \in \mathbb{R}[Z]^{t \times t}$, and matrices $C_{\ell} \in \mathbb{R}[Z]^{t \times t}, \ell=1, \ldots, m$, satisfying

(a) $D_{\ell}=C_{\ell}^{*}(-f) C_{\ell}$,

(b) for each $x \in \mathbb{R},-f(x) \succeq 0$ if and only if for all $\ell, D_{\ell}(x) \succeq 0$.

From (b) it follows that the diagonal matrix $\left[\begin{array}{ccc}-D_{1} & & \\ & \ddots & \\ & & -D_{m}\end{array}\right]$ is nowhere negative semidefinite. If $D_{\ell}$ is the diagonal matrix with entries $d_{j, \ell}, j=1, \ldots, t$, then again by [PD, Theorem 6.3.8] we deduce $-1 \in M_{\left\{d_{j, \ell} \mid j=1, \ldots, t, \ell=1, \ldots, m\right\}}$. Like in (2) we have $d_{j, \ell} \in M_{\left\{D_{\ell}\right\}} \subseteq M_{\left\{D_{1}, \ldots, D_{m}\right\}}$ for all $j, \ell$. Since $M_{\left\{D_{1}, \ldots, D_{m}\right\}} \subseteq M_{\{-f\}}$ by (a), we conclude as in (3) that $-1 \in M_{\{-f\}}$. 
Unlike in the univariate case or for Proposition 1(a) where a satisfactory statement on the level of multivariate matrix polynomials has been given in the previous subsection (see Theorem 3), there does not seem to exist a straightforward extension of Proposition [1, (b) to the multivariate case.

Example 5. Consider $f \in \operatorname{Sym} \mathbb{R}[\bar{X}]^{2 \times 2}$. We have $f \npreceq 0$ on $\mathbb{R}^{n}$ if and only if $\operatorname{tr} f(x)>0$ or $\operatorname{det} f(x)<0$ for all $x \in \mathbb{R}^{n}$.

(a) Let $f$ be diagonal. Then $f \npreceq 0$ on $\mathbb{R}^{n}$ if and only if there exist $p_{i} \in$ $\mathbb{R}[\bar{X}]^{2 \times 2}$ such that $\sum_{i} p_{i}^{*} f p_{i} \in 1+M_{\varnothing}$. Indeed, the implication $(\Leftarrow)$ is easy (cf. Proposition 1). For the converse implication, suppose $f=\left[\begin{array}{ll}a & 0 \\ 0 & c\end{array}\right] \npreceq 0$ on $\mathbb{R}^{n}$. Then $a>0$ on $S_{\{-c\}}$ and therefore $p^{2} a=1+\sigma-\tau c$ for some $p \in \mathbb{R}[\bar{X}]$ and sums of squares $\sigma, \tau \in \mathbb{R}[\bar{X}]$ by Krivine's Positivstellensatz (see, e.g. [Ma, Chapter 2] or [PD, Section 4.2]). Obviously, there exist diagonal $h_{i} \in \mathbb{R}[\bar{X}]^{2 \times 2}$ such that $\sum_{i} h_{i}^{*} f h_{i}=\left[\begin{array}{cc}p^{2} a & 0 \\ 0 & \tau c\end{array}\right]$. Now

$$
\sum_{i} h_{i}^{*} f h_{i}+\sum_{i}\left[\begin{array}{ll}
0 & 1 \\
1 & 0
\end{array}\right]^{*} h_{i}^{*} f h_{i}\left[\begin{array}{ll}
0 & 1 \\
1 & 0
\end{array}\right]=\left[\begin{array}{cc}
1+\sigma & 0 \\
0 & 1+\sigma
\end{array}\right] \in 1+M_{\varnothing}
$$

(b) If $f=\left[\begin{array}{ll}a & b \\ b & c\end{array}\right]$, then

$$
\left[\begin{array}{cc}
1 & -b \\
0 & a
\end{array}\right]^{*} f\left[\begin{array}{cc}
1 & -b \\
0 & a
\end{array}\right]+\left[\begin{array}{cc}
0 & 1 \\
-c & b
\end{array}\right]^{*} f\left[\begin{array}{cc}
0 & 1 \\
-c & b
\end{array}\right]=\left[\begin{array}{cc}
\operatorname{tr} f & 0 \\
0 & (\operatorname{tr} f)(\operatorname{det} f)
\end{array}\right] .
$$

Unable to settle the general case, we assume $\operatorname{tr} f(x) \neq 0$ for all $x \in \mathbb{R}^{n}$. Then the diagonal matrix on the right hand side of (4) is nowhere negative semidefinite on $\mathbb{R}^{n}$. By part (国) above, we obtain $h_{i} \in \mathbb{R}[\bar{X}]^{2 \times 2}$ such that $\sum_{i} h_{i}^{*} f h_{i} \in 1+M_{\varnothing}$.

Example 6. The diagonal matrix

$$
f:=\left[\begin{array}{ccc}
X_{1} & 0 & 0 \\
0 & X_{2} & 0 \\
0 & 0 & X_{1} X_{2}+1
\end{array}\right] \in \operatorname{Sym} \mathbb{R}[\bar{X}]^{3 \times 3}
$$

satisfies $f \npreceq 0$ on $\mathbb{R}^{2}$ and yet there do not exist $p_{i} \in \mathbb{R}[\bar{X}]^{2 \times 2}$ with $\sum_{i} p_{i}^{*} f p_{i} \in$ $1+M_{\varnothing}$. This example is inspired by [Ma, Example 7.3.2(i)] which is a modification of the Jacobi-Prestel example [PD, Example 6.3.1].

By way of contradiction, assume $\sum_{i} p_{i}^{*} f p_{i}=1+q$ where $q \in M_{\varnothing}$. Extracting the top left entry on both sides of this equation, we get sums of squares $\sigma_{1}, \sigma_{2}, \sigma_{3}, \tau \in \mathbb{R}\left[X_{1}, X_{2}\right]$ with

$$
\sigma_{1} X_{1}+\sigma_{2} X_{2}+\sigma_{3}\left(X_{1} X_{2}+1\right)=1+\tau \text {. }
$$

In particular, -1 lies in the quadratic module generated in $\mathbb{R}\left[X_{1}, X_{2}\right]$ by $-X_{1}$, $-X_{2}$ and $-X_{1} X_{2}-1$, contradicting the existence of a semiordering in $\mathbb{R}\left[X_{1}, X_{2}\right]$ containing these three polynomials (cf. [Ma, Example 7.3.1]).

We will see that a sum of hermitian squares representation with denominators (and weights) does exist for matrix polynomials nonnegative on a compact set 
with archimedean corresponding quadratic module (see Subsection 3.4). This seems to mimic the situation for polynomials in noncommutative variables studied e.g. in [KS], where a Nirgendsnegativsemidefinitheitsstellensatz describing nonnegativity on a bounded set has been given, while the global case is still an open problem; see [KS, Open problem 3.2] for a precise formulation.

\section{ARChimedeAn QUADRATIC MODULES OF MATRIX POLYNOMIALS}

$C^{*}$-algebras $\mathcal{A}$ enjoy the following boundedness property: for all $a \in \mathcal{A}$ there is an $N \in \mathbb{N}$ such that $N-a^{*} a$ is a sum of hermitian squares (actually a hermitian square). In this section, we try to mimic this boundedness property in an algebraic context for other rings with involution. In the rigid context of (matrix) polynomials, sums of hermitian squares have of course to be replaced by a general quadratic module.

3.1. Archimedean quadratic modules. A quadratic module $M$ of a ring with involution $\mathcal{A}$ is said to be archimedean if

$$
\forall a \in \mathcal{A} \exists N \in \mathbb{N}: N-a^{*} a \in M .
$$

To a quadratic module $M \subseteq \operatorname{Sym} \mathcal{A}$ we associate its ring of bounded elements

$$
H_{M}(\mathcal{A}):=\left\{a \in \mathcal{A} \mid \exists N \in \mathbb{N}: N-a^{*} a \in M\right\} .
$$

A quadratic module $M \subseteq \operatorname{Sym} \mathcal{A}$ is thus archimedean if and only if $H_{M}(\mathcal{A})=\mathcal{A}$. The name ring of bounded elements is justified by the following proposition originally due to Vidav [Vi]; see also Ci] for a more accessible reference:

Proposition 7 (Vidav). Let $\mathcal{A}$ be a ring with involution, $\frac{1}{2} \in \mathcal{A}$ and $M \subseteq$ $\operatorname{Sym} \mathcal{A}$ a quadratic module. Then $H_{M}(\mathcal{A})$ is a subring of $\mathcal{A}$ and is closed under the involution.

In case $\mathcal{A}$ is an $\mathbb{R}$-algebra, it suffices to check the archimedean condition (5) on a set of algebra generators.

Lemma 8. A quadratic module $M \subseteq \operatorname{Sym} \mathbb{R}[\bar{X}]^{t \times t}$ is archimedean if and only if there exists $N \in \mathbb{N}$ with $N-\sum_{i} X_{i}^{2} \in M$.

Proof. The "only if" direction is obvious. For the converse, observe that $\mathbb{R}[\bar{X}]^{t \times t}$ is generated as an $\mathbb{R}$-algebra by $\bar{X}$ and the matrix units $E_{i j}, i, j=1, \ldots, t$. By assumption,

$$
N-X_{j}^{2}=\left(N-\sum_{i} X_{i}^{2}\right)+\sum_{i \neq j} X_{i}^{2} \in M,
$$

so $X_{j} \in H_{M}\left(\mathbb{R}[\bar{X}]^{t \times t}\right)$ for every $j$. On the other hand, $E_{i j}^{*} E_{i j}=E_{j j}$ and thus

$$
1-E_{i j}^{*} E_{i j}=\sum_{k \neq j} E_{k k}^{*} E_{k k} \in M
$$

Hence by Proposition 7, $H_{M}\left(\mathbb{R}[\bar{X}]^{t \times t}\right)=\mathbb{R}[\bar{X}]^{t \times t}$ so $M$ is archimedean. 
3.2. Pure states. In functional analysis, the concept of pure states is wellestablished, see e.g. [Ar, Sections 1.6 and 1.7] for the classical application to $C^{*}$-algebras and their representations. Here we adopt these ideas to matrix polynomials.

Let $G \subseteq \operatorname{Sym} \mathbb{R}[\bar{X}]^{t \times t}$. A linear form $L: \operatorname{Sym} \mathbb{R}[\bar{X}]^{t \times t} \rightarrow \mathbb{R}$ is called a state on $\left(\operatorname{Sym} \mathbb{R}[\bar{X}]^{t \times t}, M_{G}\right)$ if $L\left(M_{G}\right) \subseteq \mathbb{R}_{\geq 0}$ and $L(1)=1$. A state $L$ is called pure if it is an extreme point of the convex set of all states, i.e., it is not a proper convex combination of two states other than $L$.

We now come to the central result of this article. It is a matrix polynomial version of the well-known theorem stating that for every pure state $L$ on a $C^{*}$ algebra $\mathcal{A}$ there exists a unit vector $v$ in a Hilbert space $\mathcal{H}$ and an irreducible *-representation $\pi: \mathcal{A} \rightarrow \mathcal{B}(\mathcal{H})$ such that $L(a)=\langle\pi(a) v, v\rangle$ for all $a \in \mathcal{A}$ (see e.g. [Ar, Theorem 1.6.6]).

Theorem 9. Suppose $G \subseteq \operatorname{Sym} \mathbb{R}[\bar{X}]^{t \times t}$ and $M_{G}$ is archimedean. For each pure state $L$ on $\left(\operatorname{Sym} \mathbb{R}[\bar{X}]^{t \times t}, M_{G}\right)$, there exists $x \in S_{G}$ and a unit vector $v \in \mathbb{R}^{t}$ such that

$$
L(p)=\langle p(x) v, v\rangle \quad \text { for all } p \in \operatorname{Sym} \mathbb{R}[\bar{X}]^{t \times t} .
$$

Proof. We extend $L$ to $\mathbb{C}[\bar{X}]^{t \times t}$ by setting

$$
L(p+\dot{\mathrm{i}} q)=\frac{1}{2}\left(L\left(p+p^{*}\right)+\dot{\mathrm{i}} L\left(q+q^{*}\right)\right)
$$

for $p, q \in \mathbb{R}[\bar{X}]^{t \times t}$. This is the unique $\mathbb{C}$-linear extension of $L$ satisfying $L\left(f^{*}\right)=$ $L(f)^{*}$ for all $f \in \mathbb{C}[\bar{X}]^{t \times t}$. Let

$$
M_{G}^{\mathbb{C}}:=\left\{\sum_{j=1}^{N} p_{j}^{*} g_{j} p_{j} \mid N \in \mathbb{N}, g_{j} \in\{1\} \cup G, p_{j} \in \mathbb{C}[\bar{X}]^{t \times t}\right\}
$$

be the quadratic module generated by $G$ in $\mathbb{C}[\bar{X}]^{t \times t}$. Then $L$ is nonnegative on $M_{G}^{\mathbb{C}}$. Indeed, given $f=(p+\dot{\mathrm{i}} q)^{*} g(p+\dot{\mathrm{i}} q)$ with $p, q \in \mathbb{R}[\bar{X}]^{t \times t}$ and $g \in\{1\} \cup G$, we have $f=\left(p^{*} g p+q^{*} g q\right)+\dot{\mathrm{i}}\left(p^{*} g q-q^{*} g p\right)$. Applying the definition (6) of $L$, we obtain $L(f)=L\left(p^{*} g p+q^{*} g q\right) \in L\left(M_{G}\right) \subseteq \mathbb{R}_{\geq 0}$, as desired. For later use let us observe that $M_{G}^{\mathbb{C}}$ is archimedean: write $f \in \mathbb{C}[\bar{X}]^{t \times t}$ as $f=f_{1}+\dot{\mathbb{i}} f_{2}$ with $f_{j} \in \mathbb{R}[\bar{X}]^{t \times t}$. Then $f_{j} \in H\left(M_{G}\right) \subseteq H\left(M_{G}^{\mathbb{C}}\right)$ and $\dot{\mathrm{i}} \in H\left(M_{G}^{\mathbb{C}}\right)$. Hence Proposition 7 implies $H\left(M_{G}^{\mathbb{C}}\right)=\mathbb{C}[\bar{X}]^{t \times t}$.

By the Cauchy-Schwarz inequality for semi-scalar products,

$$
J:=\left\{f \in \mathbb{C}[\bar{X}]^{t \times t} \mid L\left(f^{*} f\right)=0\right\}
$$

is a linear subspace of $\mathbb{C}[\bar{X}]^{t \times t}$. Similarly, we see that

$$
\langle\bar{p}, \bar{q}\rangle:=L\left(q^{*} p\right)
$$

defines a scalar product on $\mathbb{C}[\bar{X}]^{t \times t} / J$, where $\bar{p}:=p+J$ denotes the residue class of $p \in \mathbb{C}[\bar{X}]^{t \times t}$ modulo $J$. Let $\mathcal{H}$ denote the completion of $\mathbb{C}[\bar{X}]^{t \times t} / J$ with respect to this scalar product. Note that $\mathcal{H} \neq\{0\}$ since $1 \notin J$.

We proceed to show $J$ is a left ideal of $\mathbb{C}[\bar{X}]^{t \times t}$. Let $f \in \mathbb{C}[\bar{X}]^{t \times t}$. Since $M_{G}^{\mathbb{C}}$ is archimedean, there is some $N \in \mathbb{N}$ with $N-f^{*} f \in M_{G}^{\mathbb{C}}$. Hence for all $p \in \mathbb{C}[\bar{X}]^{t \times t}$, we have

$$
0 \leq L\left(p^{*}\left(N-f^{*} f\right) p\right) \leq N L\left(p^{*} p\right) .
$$


This shows that $L\left(p^{*} f^{*} f p\right)=0$ for all $p \in J$, i.e., $f p \in J$.

Because $J$ is a left ideal, the map

$$
\pi: \mathbb{C}[\bar{X}]^{t \times t} \rightarrow \mathcal{B}(\mathcal{H}), f \mapsto(\bar{p} \mapsto \overline{f p})
$$

is well-defined. Here $\bar{p} \mapsto \overline{f p}$ stands for the unique bounded linear extension to $\mathcal{H}$ of the left multiplication with $\bar{f}$ on $\mathbb{C}[\bar{X}]^{t \times t} / J$, which is well-defined by (9). Using the definition (8) of the scalar product, it is easy to see that $\pi$ is a homomorphism of rings with involution, i.e., a $*$-representation of $\mathbb{C}[\bar{X}]^{t \times t}$ on $\mathcal{H}$. Setting $w:=\overline{1} \in \mathcal{H}$, we observe that

$$
L(f)=\langle\pi(f) w, w\rangle
$$

for all $f \in \mathbb{C}[\bar{X}]^{t \times t}$.

We claim that the commutant $\pi\left(\mathbb{C}[\bar{X}]^{t \times t}\right)^{\prime}$ of the image of $\pi$ in $\mathcal{B}(\mathcal{H})$ is $\mathbb{C}$. To see this, we take an arbitrary operator $T \in \pi\left(\mathbb{C}[\bar{X}]^{t \times t}\right)^{\prime}$. Since the commutant is closed under the involution and $T=\frac{T+T^{*}}{2}+\dot{\mathrm{i}} \frac{T-T^{*}}{2 \dot{\mathrm{i}}}$, we are reduced to the case $T=T^{*}$. By the spectral theorem, $T$ decomposes into projections belonging to $\{T\}^{\prime \prime} \subseteq \pi\left(\mathbb{C}[\bar{X}]^{t \times t}\right)^{\prime}$. So we can even assume $T$ is a projection. By way of contradiction, assume $T \neq 0$ and $T \neq 1$. Since $T \in \pi\left(\mathbb{C}[\bar{X}]^{t \times t}\right)^{\prime}$ and $w$ is a cyclic vector for $\pi$ by construction, it follows that $T w \neq 0$ and $(1-T) w \neq 0$. This allows us to define states $L_{i}$ on $\left(\operatorname{Sym} \mathbb{R}[\bar{X}]^{t \times t}, M_{G}\right)$ by

$$
L_{1}(f)=\frac{\langle\pi(f) T w, T w\rangle}{\|T w\|^{2}} \quad \text { and } \quad L_{2}(f)=\frac{\langle\pi(f)(1-T) w,(1-T) w\rangle}{\|(1-T) w\|^{2}}
$$

for all $f \in \operatorname{Sym} \mathbb{R}[\bar{X}]^{t \times t}$. One checks that $L$ is a convex combination of $L_{1}$ and $L_{2}$. The state $L$ being pure, we obtain $L=L_{i}$. By (11), this implies

$$
\langle\pi(f) w, \lambda w\rangle=\lambda\langle\pi(f) w, w\rangle=\langle\pi(f) T w, T w\rangle=\langle T \pi(f) w, T w\rangle=\langle\pi(f) w, T w\rangle
$$

for all $f \in \mathbb{C}[\bar{X}]^{t \times t}$, where $\lambda:=\|T w\|^{2}$. In particular, $T w=\lambda w$ since $w$ is a cyclic vector for $\pi$. This implies $\lambda \in\{0,1\}$ since $T$ is a projection, a contradiction.

By [La, Theorem 3.1], $\operatorname{ker} \pi=I^{t \times t}$ for an ideal $I$ of $\mathbb{C}[\bar{X}]$. Since $\operatorname{ker} \pi$ is closed under the involution, $I$ is closed under complex conjugation. Moreover $\mathbb{C}[\bar{X}] / I$ is contained in the center of $(\mathbb{C}[\bar{X}] / I)^{t \times t}=\mathbb{C}[\bar{X}]^{t \times t} / \operatorname{ker} \pi \cong \pi\left(\mathbb{C}[\bar{X}]^{t \times t}\right)$ which is $\mathbb{C}$ by the above. Hence $\mathbb{C}[\bar{X}] / I=\mathbb{C}$ and $\pi\left(\mathbb{C}[\bar{X}]^{t \times t}\right) \cong \mathbb{C}^{t \times t}$ as a $C^{*}$ algebra. In particular, there exists $x \in \mathbb{C}^{n}$ such that $I=\{p \in \mathbb{C}[\bar{X}] \mid p(x)=0\}$. Actually $x \in \mathbb{R}^{n}$ since $I=I^{*}$. Also, $\mathcal{H}=\pi\left(\mathbb{C}[\bar{X}]^{t \times t}\right) w$ is finite-dimensional.

Next we claim that $\pi$ is an irreducible $*$-representation. Indeed, suppose $U$ is a linear subspace of $\mathcal{H}$ invariant under every $\pi(f)$ for $f \in \mathbb{C}[\bar{X}]^{t \times t}$. Let $P: \mathcal{H} \rightarrow U$ denote the orthogonal projection. It suffices to show that $P \in$ $\pi\left(\mathbb{C}[\bar{X}]^{t \times t}\right)^{\prime}=\mathbb{C}$, i.e., $\pi(f) P=P \pi(f)$ for each $f \in \mathbb{C}[\bar{X}]^{t \times t}$. By the standard trick, we reduce to the case $f=f^{*}$. But then

$$
\pi(f) P=P \pi(f) P=(P \pi(f) P)^{*}=(\pi(f) P)^{*}=P \pi(f) .
$$

As indicated in the commutative diagram below, $\pi$ now induces an irreducible *-representation $\bar{\pi}$ of $\mathbb{C}^{t \times t}$. This representation is unitarily equivalent to the identity representation $\iota$ $\mathrm{Ar}$, Corollary 2 to Theorem 1.4.4], i.e., there is a unitary map $\Phi: \mathcal{H} \rightarrow \mathbb{C}^{t}$ making the diagram below commute. 


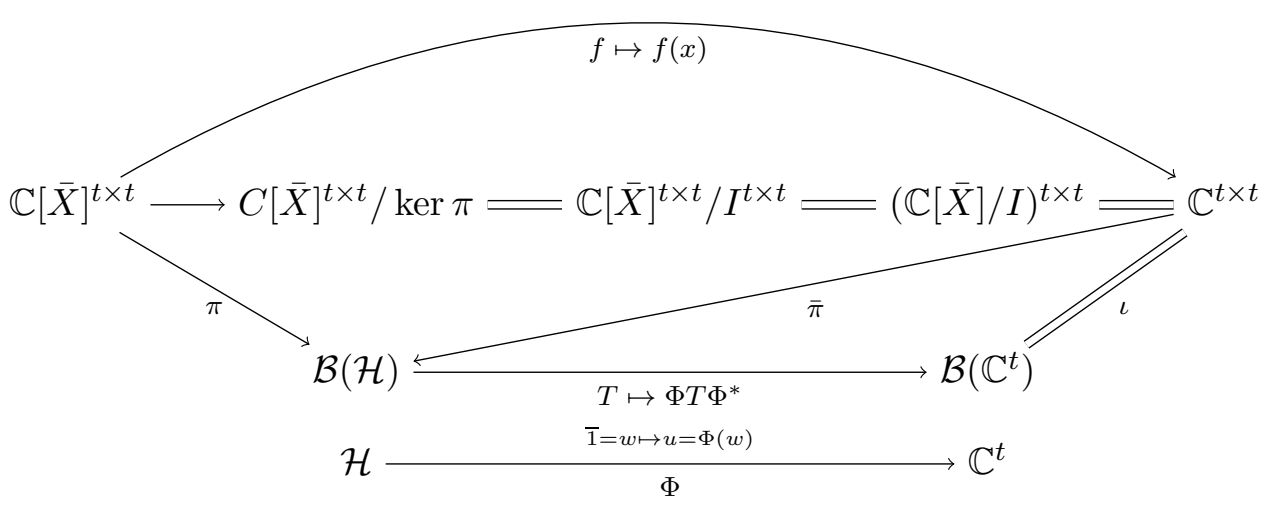

Let $u:=\Phi(w) \in \mathbb{C}^{t}$. For each $p \in \mathbb{C}[\bar{X}]^{t \times t}$, we have

$$
\begin{aligned}
L(p) & =\langle\pi(p) w, w\rangle=\langle\Phi \pi(p) w, \Phi w\rangle=\left\langle\Phi \pi(p) \Phi^{*} u, u\right\rangle \\
& =\left\langle\Phi \bar{\pi}(p(x)) \Phi^{*} u, u\right\rangle=\langle\iota(p(x)) u, u\rangle=\langle p(x) u, u\rangle .
\end{aligned}
$$

In particular, we get $\operatorname{tr}\left(A u u^{*}\right)=\langle A u, u\rangle=L(A) \in \mathbb{R}$ for all $A \in \mathbb{R}^{t \times t}$. This implies $u u^{*} \in \mathbb{R}^{t \times t}$, that is, $u u^{*}$ is a real positive semidefinite rank one matrix and can thus be factorized as $u u^{*}=v v^{*}$ for some $v \in \mathbb{R}^{t}$. We can now rewrite (12) as

$$
L(p)=\langle p(x) u, u\rangle=\operatorname{tr}\left(p(x) u u^{*}\right)=\operatorname{tr}\left(p(x) v v^{*}\right)=\langle p(x) v, v\rangle
$$

for all $p \in \mathbb{R}[\bar{X}]^{t \times t}$. Also note that $\langle v, v\rangle=L(1)=1$, i.e., $v$ is a unit vector.

It remains to show that $x \in S_{G}$. To show this, let $g \in G$ and $z \in \mathbb{R}^{t}$. Choose $A \in \mathbb{R}^{t \times t}$ with $z=A v$. Then

$$
\langle g(x) z, z\rangle=\langle g(x) A v, A v\rangle=\left\langle A^{*} g(x) A v, v\right\rangle=L\left(A^{*} g A\right) \geq 0
$$

since $A^{*} g A \in M_{G}$.

Proposition 10. Let $G \subseteq \operatorname{Sym} \mathbb{R}[\bar{X}]^{t \times t}$. For each $x \in S_{G}$ and each unit vector $v \in \mathbb{R}^{t}$, the state $L$ on $\left(\operatorname{Sym} \mathbb{R}[\bar{X}]^{t \times t}, M_{G}\right)$ defined by $L(p)=\langle p(x) v, v\rangle$ is pure.

Proof. For convenience of notation, set $L_{0}:=L$. Suppose there are states $L_{1}$ and $L_{2}$ on $\left(\operatorname{Sym} \mathbb{R}[\bar{X}]^{t \times t}, M_{G}\right)$ such that $2 L_{0}=L_{1}+L_{2}$. Extending each $L_{i}$ to $\mathbb{C}[\bar{X}]^{t \times t}$ as in ([6), this equality still holds. Define linear subspaces $J_{i} \subseteq \mathbb{C}[\bar{X}]^{t \times t}$ as in (77) by $J_{i}:=\left\{f \in \mathbb{C}[\bar{X}]^{t \times t} \mid L_{i}\left(f^{*} f\right)=0\right\}$. Obviously

$$
J_{1} \cap J_{2}=J_{0}=\left\{f \in \mathbb{C}[\bar{X}]^{t \times t} \mid f(x) v=0\right\} .
$$

In particular, $\mathbb{C}[\bar{X}]^{t \times t} / J_{0} \cong \mathbb{C}^{t}$ as vector spaces. Hence $\mathcal{H}_{i}:=\mathbb{C}[\bar{X}]^{t \times t} / J_{i}$ is of dimension at most $t$. The GNS construction for $L_{i}$ yields a scalar product $\langle.,\rangle_{i}$ on $\mathcal{H}_{i}$ defined as in (8) and a $*$-representation $\pi_{i}: \mathbb{C}[\bar{X}]^{t \times t} \rightarrow \mathcal{B}\left(\mathcal{H}_{i}\right)$ (cf. the proof of the previous theorem). Again by [La, Theorem 3.1], there are ideals $I_{i} \subsetneq \mathbb{C}[\bar{X}]$ such that ker $\pi_{i}=I_{i}^{t \times t}$ and therefore $\mathbb{C}[\bar{X}]^{t \times t} / \operatorname{ker} \pi_{i} \cong\left(\mathbb{C}[\bar{X}] / I_{i}\right)^{t \times t}$. In particular,

$$
\operatorname{dim} \mathcal{B}\left(\mathcal{H}_{i}\right) \geq \operatorname{dim}\left(\mathbb{C}[\bar{X}]^{t \times t} / \operatorname{ker} \pi_{i}\right) \geq t^{2} \operatorname{dim}\left(\mathbb{C}[\bar{X}] / I_{i}\right) \geq t^{2}
$$


whence $\operatorname{dim} \mathcal{H}_{i} \geq t$ and therefore $\operatorname{dim} \mathcal{H}_{i}=t$. Now by (13), we have $J_{0}=J_{1}=$ $J_{2}$. Therefore we have three scalar products on $\mathcal{H}:=\mathcal{H}_{0}=\mathcal{H}_{1}=\mathcal{H}_{2}$, and we find positive definite matrices $G_{1}, G_{2} \in \mathbb{C}^{t \times t}$ such that

$$
L_{i}\left(q^{*} p\right)=\langle\bar{p}, \bar{q}\rangle_{i}=v^{*} q(x)^{*} G_{i} p(x) v
$$

where $\bar{p}=p(x) v$ denotes the residue class of $p$ modulo $J_{i}$ (this is also true for $i=0$ with $G_{0}$ being the identity matrix). Since

$$
v^{*} C^{*} G_{i} A B v=\langle\overline{A B}, \bar{C}\rangle_{i}=L_{i}\left(C^{*} A B\right)=\left\langle\bar{B}, \overline{A^{*} C}\right\rangle_{i}=v^{*} C^{*} A G_{i} B v
$$

for all $A, B, C \in \mathbb{C}^{t \times t}$, it follows that $G_{i} A=A G_{i}$ for all $A \in \mathbb{C}^{t \times t}$, i.e., $G_{i} \in \mathbb{C}$. More precisely, $G_{i}=G_{i}\langle v, v\rangle=v^{*} G_{i} v=L_{i}(1)=1$. Thus $L=L_{1}=L_{2}$.

For $0 \neq v \in \mathbb{R}^{t}$, denote by $[v]$ the linear subspace spanned by $v$ seen as an element of the real projective space $\mathbb{P}^{t-1}(\mathbb{R})$ of dimension $t-1$.

Corollary 11. Suppose $G \subseteq \operatorname{Sym} \mathbb{R}[\bar{X}]^{t \times t}$ and $M_{G}$ is archimedean. We have a bijection between $S_{G} \times \mathbb{P}^{t-1}(\mathbb{R})$ and the set of pure states on $\left(\operatorname{Sym} \mathbb{R}[\bar{X}]^{t \times t}, M_{G}\right)$ well-defined by associating to each $(x,[v]) \in S_{G} \times \mathbb{P}^{t-1}(\mathbb{R}), v$ a unit vector of $\mathbb{R}^{t}$, the map $p \mapsto\langle p(x) v, v\rangle$.

Proof. By Proposition 10 and $\langle p(x) v, v\rangle=\langle p(x)(-v),-v\rangle$, the map is welldefined. It is surjective by Theorem [9. To show that it is injective, let $(x,[v]),(y,[w]) \in S_{G} \times \mathbb{P}^{t-1}(\mathbb{R})$ with unit vectors $v, w \in \mathbb{R}^{t}$ satisfy

$$
\langle p(x) v, v\rangle=\langle p(y) w, w\rangle
$$

for all $p \in \operatorname{Sym} \mathbb{R}[\bar{X}]^{t \times t}$. Using (14) with $p=E_{i j}+E_{j i}$ yields $v v^{*}=w w^{*}$. Then $[v]=[w]$ since $\left(v^{*} w\right) v=v v^{*} w=w w^{*} w=w$. Setting $p=X_{i}$ in (14), we get moreover $x_{i}=y_{i}$ whence $x=y$.

In general, Theorem 9 and Corollary 11 fail badly for nonarchimedean $M_{G}$.

Example 12. Take $G=\varnothing, t=1$ and $n=2$, i.e., consider pure states on $\left(\mathbb{R}[X, Y], M_{\varnothing}\right)$ where $X$ and $Y$ are two variables and $M_{\varnothing}$ is the cone of sums of squares of polynomials. We endow the (algebraic) dual $\mathbb{R}[X, Y]^{\vee}$ with the weak* topology and consider the closed convex cone $M_{\varnothing}^{\vee} \subseteq \mathbb{R}[X, Y]^{\vee}$ of linear forms $L: \mathbb{R}[X, Y] \rightarrow \mathbb{R}$ with $L\left(M_{\varnothing}\right) \subseteq \mathbb{R}_{\geq 0}$. Note that each $0 \neq L \in M_{\varnothing}^{\vee}$ becomes a state on $\left(\mathbb{R}[X, Y], M_{\varnothing}\right)$ after multiplication with a positive scalar (for $L(1)=0$ implies $L=0$ by a Cauchy-Schwarz argument). Choose a polynomial $f$ with $f \geq 0$ on $\mathbb{R}^{2}$ and $f \notin M_{\varnothing}$, for instance the Motzkin polynomial $f=X^{2} Y^{4}+$ $X^{4} Y^{2}-3 X^{2} Y^{2}+1$ [Ma, Proposition 1.2.2]. Since $M_{\varnothing}$ is closed in $\mathbb{R}[X, Y]$ with respect to the finest locally convex topology (see, e.g., [Ma, Proposition 4.1.2(2)] together with [Ma, Example 4.1.5]), the Hahn-Banach separation theorem [Ba, Theorem III.3.4] yields $L_{0} \in M_{\varnothing}^{\vee}$ with $L_{0}(f)<0$. As explained above, we can assume that $L_{0}$ is a state on $\left(\mathbb{R}[X, Y], M_{\varnothing}\right)$.

Fix a double sequence $\left(c_{i j}\right)_{i, j \in \mathbb{N}_{0}}$ of $c_{i j}>0$ satisfying $\sum_{i, j} c_{i j} L_{0}\left(X^{2 i} Y^{2 j}\right)=1$. Now set

$$
C:=\left\{L \in M_{\varnothing}^{\vee} \mid \sum_{i, j \in \mathbb{N}_{0}} c_{i j} L\left(X^{2 i} Y^{2 j}\right) \leq 1\right\} .
$$




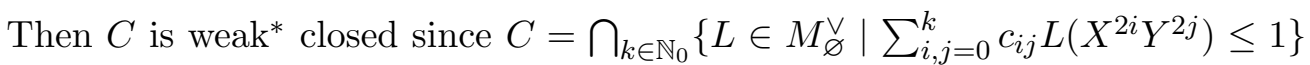
and compact (for if $L \in C$, then $\left|L\left(X^{2 i} Y^{2 j}\right)\right| \leq \frac{1}{c_{i j}}$ for all $i, j \in \mathbb{N}_{0}$, and this implies by a Cauchy-Schwarz argument similar a priori bounds for the values of $L$ on the other polynomials). In addition, both $C$ and $M_{\varnothing}^{\vee} \backslash C$ are obviously convex. Hence $C$ is a cap of $M_{\varnothing}^{\vee}$ containing $L_{0}$ (see [Ph, page 80]).

By the Krein-Milman theorem [Ba, Theorem III.4.1], there exists an extreme point $L$ of $C$ such that $L(f)<0$. By Choquet theory [Ph, Proposition 13.1], $L$ lies on an extreme ray of $M_{\varnothing}^{\vee}$. After normalization, $L$ is a pure state on $\left(\mathbb{R}[X, Y], M_{\varnothing}\right)$. Since $L(f)<0$ and $f \geq 0$ on $\mathbb{R}^{2}, L$ cannot be a point evaluation.

3.3. Positive semidefinite matrix polynomials. Now we are ready to give a version of Proposition 1(国) for matrix polynomials in the archimedean case, originally due to $\mathrm{Hol}$ and Scherer $\mathrm{HS}$, Corollary 1]. Using the above classification of pure states, the proof reduces to an easy separation argument. In contrast to this, the original proof of $\mathrm{Hol}$ and Scherer is more involved.

Theorem 13 (Hol \& Scherer). Suppose $G \cup\{f\} \subseteq \operatorname{Sym} \mathbb{R}[\bar{X}]^{t \times t}$ and $M_{G}$ is archimedean. If $f \succ 0$ on $S_{G}$, then $f \in M_{G}$.

Proof. In the terminology of Barvinok [Ba, Definition III.1.6], Proposition 7 together with the identity $4 s=(s+1)^{2}-(s-1)^{2}$ shows that $M_{G}$ is an archimedean quadratic module if and only if 1 is an algebraic interior point of the convex cone $M_{G} \subseteq \operatorname{Sym} \mathbb{R}[\bar{X}]^{t \times t}$. Recall: $f$ is an algebraic interior point of $M_{G}$ if for every $p \in \operatorname{Sym} \mathbb{R}[\bar{X}]^{t \times t}$ there exists $\varepsilon>0$ with $f+\varepsilon p \in M_{G}$.

Suppose $f \notin M_{G}$. We will find $x \in S_{G}$ such that $f(x) \nsucc 0$. The existence of an algebraic interior point of $M_{G}$ allows us to separate the convex sets $M_{G}$ and $\mathbb{R}_{>0} f$ by the Eidelheit-Kakutani separation theorem [Ba, Theorem III.1.7]. More precisely, there exists a state $L$ on $\left(\operatorname{Sym} \mathbb{R}[\bar{X}]^{t \times t}, M_{G}\right)$ with $L(f) \leq 0$. The set of all such states is weak* compact by Tikhonov's theorem (cf. the proof of Alaoglu's theorem [Ba, Theorem III.2.9]). Hence by the Krein-Milman theorem [Ba, Theorem III.4.1], $L$ can be chosen to be pure.

By Theorem 9, there exists $x \in S_{G}$ and a unit vector $v \in \mathbb{R}^{t}$ such that $L(p)=\langle p(x) v, v\rangle$ for all $p \in \operatorname{Sym} \mathbb{R}[\bar{X}]^{t \times t}$. In particular, $\langle f(x) v, v\rangle=L(f) \leq 0$ as desired.

Corollary 14. Suppose $G \subseteq \operatorname{Sym} \mathbb{R}[\bar{X}]^{t \times t}$ and $M_{G}$ is archimedean. For $f \in$ Sym $\mathbb{R}[\bar{X}]^{t \times t}$, the following are equivalent:

(i) $f \succeq 0$ on $S_{G}$;

(ii) $f+\varepsilon \in M_{G}$ for all $\varepsilon \in \mathbb{R}_{>0}$.

For $t=1$, Theorem 13 specializes to Putinar's Positivstellensatz $\mathrm{Pu}$. To avoid possible confusion, we use the letter $Q$ to denote quadratic modules in commutative rings with trivial involution. For instance, if $G \subseteq \mathbb{R}[\bar{X}]$ we denote the quadratic module generated by $G$ in $\mathbb{R}[\bar{X}]$ by $Q_{G}$, i.e.,

$$
Q_{G}=\left\{\sum_{i=1}^{N} p_{i}^{2} g_{i} \mid N \in \mathbb{N}, g_{i} \in\{1\} \cup G, p_{i} \in \mathbb{R}[\bar{X}]\right\} .
$$

Note that $Q_{G}=M_{G}$ for $t=1$ but $Q_{G} \subsetneq M_{G}$ for $t>1$. 
Corollary 15 (Putinar). Suppose $G \cup\{f\} \subseteq \mathbb{R}[\bar{X}]$ and $Q_{G}$ is archimedean. If $f>0$ on $S_{G}$, then $f \in Q_{G}$.

Clearly, if $Q_{G}$ is archimedean then $S_{G}$ is compact. The converse is false even for finite $G \subseteq \mathbb{R}[\bar{X}]$ as shown by the Jacobi-Prestel example [PD, Example 6.3.1]. Nevertheless, there is an intimate connection between compactness and the archimedean property established by Schmüdgen Sm1. To describe his result, we introduce the following notation: Given a set $G=\left\{g_{1}, \ldots, g_{m}\right\} \subseteq$ $\mathbb{R}[\bar{X}]$ of $m$ distinct polynomials, let $\widehat{G}=\left\{g_{1}^{\delta_{1}} \cdots g_{m}^{\delta_{m}} \mid 0 \neq \delta \in\{0,1\}^{m}\right\}$ denote the set of the $2^{m}-1$ nontrivial products of the $g_{i}$.

Theorem 16 (Schmüdgen). Suppose $G \subseteq \mathbb{R}[\bar{X}]$ is finite. Then $S_{G}$ is compact if and only if the (multiplicative) quadratic module $Q_{\widehat{G}}$ is archimedean.

As an important special case, we obtain that for a singleton $G=\{g\} \subseteq \mathbb{R}[\bar{X}]$, $S_{G}$ is compact if and only if $Q_{G}$ is archimedean. This continues to hold if $G$ has exactly two elements [PD, Corollary 6.3.7]. For this and other nontrivial strengthenings of Schmüdgen's theorem due to Jacobi and Prestel we refer to [PD, Chapter 6]. These results allow us to deduce that $M_{G}$ is archimedean in such cases by the following proposition.

Proposition 17. If $G \subseteq \mathbb{R}[\bar{X}]$, then $Q_{G}$ is archimedean if and only if $M_{G}$ is archimedean.

Proof. To prove the nontrivial direction, suppose that $M_{G}$ is archimedean, i.e., $N-\sum_{i=1}^{n} X_{i}^{2}=\sum_{j} p_{j}^{*} p_{j} g_{j}$ for some $N \in \mathbb{N}, p_{j} \in \mathbb{R}[\bar{X}]^{t \times t}$ and $g_{j} \in G \cup\{1\}$. Since the trace of a hermitian square $p_{j}^{*} p_{j}$ is a sum of squares in $\mathbb{R}[\bar{X}]$, it follows that $N-\sum_{i=1}^{n} X_{i}^{2}=\frac{1}{t} \sum_{j} \operatorname{tr}\left(p_{j}^{*} p_{j}\right) g_{j} \in Q_{G}$. Hence $Q_{G}$ is archimedean by Lemma 8 .

There does not seem to exist a viable generalization of Schmüdgen's theorem for general finite $G \subseteq \operatorname{Sym} \mathbb{R}[\bar{X}]^{t \times t}$. It does not make sense to consider $\widehat{G}$ because products of positive semidefinite matrices are not symmetric in general, let alone positive semidefinite. If $G$ is a singleton, we have $\widehat{G}=G$ but still $S_{G}$ compact does not imply $M_{G}$ archimedean.

Example 18. Let $f \in \operatorname{Sym} \mathbb{R}[\bar{X}]^{3 \times 3}$ be the diagonal matrix from Example 6 . Then $S_{\{-f\}}=\varnothing$ is compact but $M_{\{-f\}}$ is not archimedean. Otherwise Theorem 13 would imply $-1 \in M_{\{-f\}}$ which is not the case as seen in Example 6.

We now briefly turn to positivity of not necessarily symmetric matrix polynomials. For this we need the following classical lemma [Br, Section 6.3] (see also [Sw, Theorem 5.3]).

Lemma 19 (Brumfiel). Let $\mathcal{R}$ be a commutative $\mathbb{Q}$-algebra and $Q \subseteq \mathcal{R}$ a quadratic module. Then $H_{Q}(\mathcal{R})$ is integrally closed in $\mathcal{R}$.

Let $G \subseteq \mathbb{R}[\bar{X}]$ and $f \in \mathbb{R}[\bar{X}]^{t \times t}$. The quadratic module generated by $G$ in the commutative ring $\mathbb{R}[\bar{X}, f]$ endowed with the trivial involution will be denoted by $Q_{G}^{f}$. Observe that $Q_{G}^{f} \subseteq M_{G}$ if and only if $f=f^{*}$. 
Theorem 20. Suppose $G \subseteq \mathbb{R}[\bar{X}], Q_{G}$ is archimedean and $f \in \mathbb{R}[\bar{X}]^{t \times t}$. If for all $x \in S_{G}$, all real eigenvalues of $f(x)$ are positive, then $f \in Q_{G}^{f}$.

Proof. Let $q_{f} \in \mathbb{R}(\bar{X})[Y]$ be the minimal polynomial of the matrix $f$. Note that $q_{f} \in \mathbb{R}[\bar{X}, Y]$ by Gauß' lemma since $q_{f}$ divides the (monic) characteristic polynomial of $f$ by the Cayley-Hamilton theorem.

Now

$$
Y>0 \quad \text { on } \quad\left\{(x, y) \in S_{G} \times \mathbb{R} \mid q_{f}(x, y)=0\right\}=S_{G \cup\left\{q_{f},-q_{f}\right\}} .
$$

We claim that $Q_{G \cup\left\{q_{f},-q_{f}\right\}}=Q_{G}^{Y}+\mathbb{R}[\bar{X}, Y] q_{f}$ is archimedean, or equivalently, the quadratic module $Q_{G}^{f}$ is archimedean in $\mathbb{R}[\bar{X}, f]=\mathbb{R}[\bar{X}, Y] /\left(q_{f}\right)$. Indeed, since $f$ is integral over $\mathbb{R}[\bar{X}]$ and $H_{Q_{G}^{f}}(\mathbb{R}[\bar{X}, f]) \supseteq H_{Q_{G}}(\mathbb{R}[\bar{X}])=\mathbb{R}[\bar{X}]$ is integrally closed, we have $H_{Q_{G}^{f}}(\mathbb{R}[\bar{X}, f])=\mathbb{R}[\bar{X}, f]$.

By Corollary 15, $Y \in Q_{G \cup\left\{q_{f},-q_{f}\right\}}$. Plugging in $f$ for $Y$ yields $f \in Q_{G}^{f}$.

Corollary 21. Suppose $G \subseteq \mathbb{R}[\bar{X}]$ and $Q_{G}$ is archimedean. For $f \in \mathbb{R}[\bar{X}]^{t \times t}$, the following are equivalent:

(i) for all $x \in S_{G}$, all real eigenvalues of $f(x)$ are nonnegative;

(ii) $f+\varepsilon \in Q_{G}^{f}$ for all $\varepsilon \in \mathbb{R}_{>0}$.

Proof. (i) $\Rightarrow$ (ii) follows from Theorem 20, For the converse, it suffices to observe that for $p \in Q_{G}^{p}$, all real eigenvalues of $p(x)$ are nonnegative for all $x \in S_{G}$. Indeed, suppose $p=\sum_{j} h_{j}(\bar{X}, p)^{2} g_{j}$ for finitely many $g_{j} \in G \cup\{1\}$ and $h_{j} \in$ $\mathbb{R}[\bar{X}, Y]$. Let $\lambda \in \mathbb{R}, 0 \neq v \in \mathbb{R}^{t}$ and $p(x) v=\lambda v$. Then

$$
\lambda v=p(x) v=\sum_{j} h_{j}(x, p(x))^{2} g_{j}(x) v=\sum_{j} h_{j}(x, \lambda)^{2} g_{j}(x) v
$$

whence $\lambda=\sum_{j} h_{j}(x, \lambda)^{2} g_{j}(x) \geq 0$.

3.4. Matrix polynomials not negative semidefinite. We conclude this article with an application of Theorem 13 yielding a version of Proposition 1(b) for matrix polynomials.

Corollary 22 (Matrizenpolynomnirgendsnegativsemidefinitheitsstellensatz). Suppose $G \subseteq \operatorname{Sym} \mathbb{R}[\bar{X}]^{t \times t}$ and $M_{G}$ is archimedean. For $f \in \operatorname{Sym} \mathbb{R}[\bar{X}]^{t \times t}$, the following are equivalent:

(i) $f \npreceq 0$ on $S_{G}$;

(ii) there exist $p_{i} \in \mathbb{R}[\bar{X}]^{t \times t}$ such that

$$
\sum_{i} p_{i}^{*} f p_{i} \in 1+M_{G}
$$

Proof. (ii) $\Rightarrow$ (i) is immediate from Proposition 1(b). For the converse, note that $f \npreceq 0$ on $S_{G}$ if and only if $S_{G \cup\{-f\}}=\varnothing$. In this case, $-1 \succ 0$ on $S_{G \cup\{-f\}}$. Since $M_{G}$ and therefore $M_{G \cup\{-f\}}$ is archimedean, Theorem 13 implies that $-1 \in M_{G \cup\{-f\}}$ which is exactly what we need.

Acknowledgments. We thank Ronan Quarez for interesting discussions which led us to the discovery of Theorem 4 . We also appreciate the anonymous referee for his careful reading of the manuscript and his corrections. 


\section{REFERENCES}

[Ar] W. Arveson: An invitation to $C^{*}$-algebras, Graduate Texts in Mathematics 39, Springer 1976

[AIP] E.M. Aylward, S.M. Itani, P.A. Parrilo: Explicit SOS decompositions of univariate polynomial matrices and the Kalman-Yakubovich-Popov lemma, Proceedings of the 46th IEEE Conference on Decision and Control 2007, 5660-5665

[Ba] A. Barvinok: A course in convexity, Graduate Studies in Mathematics 54, AMS 2002

[Br] G.W. Brumfiel: Partially ordered rings and semi-algebraic geometry, London Mathematical Society Lecture Note Series 37, Cambridge University Press 1979

[BSS] S. Burgdorf, C. Scheiderer, M. Schweighofer: Pure states, nonnegative polynomials and sums of squares, preprint, http://arxiv.org/abs/0905.4161

[CLR] M.D. Choi, T.Y. Lam, B. Reznick: Real zeros of positive semidefinite forms I, Math. Z. 171 (1980), no. 1, 1-26

[Ci] J. Cimprič: A representation theorem for Archimedean quadratic modules on $*$-rings, Canad. Math. Bull. 52 (2009), no. 1, 39-52

[Dj1] D.Ž. Djoković: Hermitian matrices over polynomial rings, J. Algebra 43 (1976), no. 2, 359-374

[Dj2] D.Ž. Djoković: Positive semi-definite matrices as sums of squares, Linear Algebra Appl. 14 (1976), no. $1,37-40$

[FRS] J.F. Fernando, J.M. Ruiz, C. Scheiderer: Sums of squares of linear forms, Math. Res. Lett. 13 (2006), no. 5-6, 947-956

[GKS] I. Gohberg, M.A. Kaashoek, I.M. Spitkovsky: An overview of matrix factorization theory and operator applications, Factorization and integrable systems, 1-102, Oper. Theory Adv. Appl. 141, Birkhäuser 2003

[GLR] I. Gohberg, P. Lancaster, L. Rodman: Matrix polynomials, Computer Science and Applied Mathematics, Academic Press 1982

[GR] D. Gondard, P. Ribenboim: Le 17e problème de Hilbert pour les matrices, Bull. Sci. Math. (2) 98 (1974), no. 1, 49-56

[HM] J.W. Helton, S.A. McCullough: A Positivstellensatz for non-commutative polynomials, Trans. Amer. Math. Soc. 356 (2004), no. 9, 3721-3737

[HiN] C.J. Hillar, J. Nie: An elementary and constructive solution to Hilbert's 17th problem for matrices, Proc. Amer. Math. Soc. 136 (2008), no. 1, 73-76

[HS] C.W.J. Hol, C.W. Scherer: Matrix sum-of-squares relaxations for robust semi-definite programs, Math. Program. 107 (2006), no. 1-2, Ser. B, 189-211

[Ja] V.A. Jakubovič: Factorization of symmetric matrix polynomials, Dokl. Akad. Nauk SSSR 194 (1970), 532-535

[KS] I. Klep, M. Schweighofer: A Nichtnegativstellensatz for polynomials in noncommuting variables, Israel J. Math. 161 (2007), no. 1, 17-27

[La] T.Y. Lam: A first course in noncommutative rings, Graduate Texts in Mathematics 131, Springer 1991

[Ma] M. Marshall: Positive polynomials and sums of squares, Mathematical Surveys and Monographs 146, AMS 2008

[Ph] R.R. Phelps: Lectures on Choquet's theorem, Lecture Notes in Mathematics 1757, Springer 2001.

[PD] A. Prestel, C.N. Delzell: Positive polynomials. From Hilbert's 17th problem to real algebra, Springer Monographs in Mathematics 2001

[PS] C. Procesi, M. Schacher: A non-commutative real Nullstellensatz and Hilbert's 17th problem, Ann. of Math. (2) 104 (1976), no. 3, 395-406

$[\mathrm{Pu}] \quad \mathrm{M}$. Putinar: Positive polynomials on compact semi-algebraic sets, Indiana Univ. Math. J. 42 (1993), no. 3, 969-984

[RR] M. Rosenblum, J. Rovnyak: The factorization problem for nonnegative operator valued functions, Bull. Amer. Math. Soc. 77 (1971), 287-318

[Sm1] K. Schmüdgen: The $K$-moment problem for compact semi-algebraic sets, Math. Ann. 289 (1991), no. 2, 203-206 
[Sm2] K. Schmüdgen: Noncommutative real algebraic geometry - some basic concepts and first ideas, In: Putinar, Mihai (ed.) et al., Emerging applications of algebraic geometry. Papers of the IMA workshops Optimization and control, Springer. The IMA Volumes in Mathematics and its Applications 149 (2009), 325-350

[Sw] M. Schweighofer: Iterated rings of bounded elements and generalizations of Schmüdgen's Positivstellensatz, J. reine angew. Math. 554 (2003), 19-45

[Vi] I. Vidav: On some *-regular rings, Acad. Serbe Sci. Publ. Inst. Math. 13 (1959), 73-80

Igor Klep, Univerza V Mariboru, Fakulteta Za naravoslovje in matematiko, KorošKa 160, Maribor, Slovénie, and Univerza V Luubluani, FAKulteta Za matematiko in fiziko, Jadranska 21, 1111 Ljubluana, Slovénie

E-mail address: igor.klep@fmf.uni-lj.si

Markus Schweighofer, Université de Rennes 1, Laboratoire de Mathématiques, Campus de Beaulieu, 35042 Rennes Cedex, France

E-mail address: markus.schweighofer@univ-rennes1.fr 